Research Paper:

\title{
Mindfulness and Its Predictors in Women With Polycystic Ovary Syndrome
}

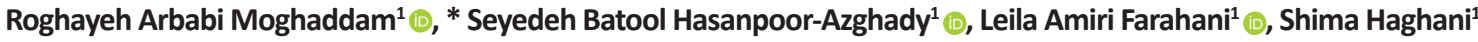

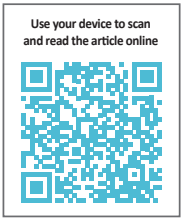

Citation Arbabi Moghaddam R, Hasanpoor-Azghady SB, Amiri Farahani L, Haghani S. [Mindfulness and Its Predictors in Women With Polycystic Ovary Syndrome (Persian)]. Journal of Inflammatory Diseases. 2020; 24(3):246-257. https://doi.org/10.32598/ JQUMS.24.3.5

doi https://doi.org/10.32598/JQUMS.24.3.5

Received: 11 Mar 2020

Accepted: 26 Jul 2020

Available Online: 01 Aug 2020
Keywords:

Mindfulness, Predictors, Polycystic ovary syndrome

\section{ABSTRACT}

Background Polycystic Ovary Syndrome (PCOS) is the most common endocrine disorder in women of reproductive age which can cause many problems such as hyperandrogenic symptoms and fertility problems. Objective The present study aimed to determine the relationship of mindfulness with hyperandrogenic symptoms and demographic and fertility factors in women with PCOS.

Methods This descriptive correlational study was conducted on 181 women with PCOS referred to Firoozabadi and Firoozgar hospitals in Tehran, Iran who were selected using a continuous sampling method and based on inclusion criteria from June 2018 to August 2019. Data were collected using a demographic/fertility form, the modified Ferriman-Gallwey Scale, Ludwig Hair Loss Scale, and Mindfulness Attention Awareness Scale (MAAS). Data were analyzed using independent t-test, one-way ANOVA, Kruskal-Wallis test, Pearson correlation test, and multiple linear regression analysis.

Findings The mean MAAS score of women was $68.61 \pm 9.88$ and was significantly correlated with age $(P=0.01)$, wife's education ( $P=0.001)$, wife's occupation $(P=0.005)$, economic status $(P=0.02)$, husband satisfaction with wife's body and appearance $(P=0.02)$, body mass index $(P=0.01)$, and duration of marriage $(P<0.001)$. According to the multiple linear regression model, the duration of marriage could predict $22 \%$ of the variance in overall MAAS score.

Conclusion Mindfulness is associated with some demographic variables, among which the marriage is its predictor. It is recommended to pay attention to the reported variables in preparation of counseling or educational programs, along with other treatments, for women with PCOS.

\section{Extended Abstract}

\section{Introduction}

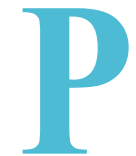

olycystic Ovary Syndrome (PCOS) is the most common endocrine disorder in women of reproductive age. The prevalence of this syndrome is about $5 \%-10 \%$ [1]. The hyperandrogenic symptoms of this syndrome, and the feeling of imperfection and failure that some of these patients have due to infertility can affect the quality of life [6] and self-satisfaction of these patients [7]. Most women with PCOS are overweight [9]. Various factors can affect the reduction of obesity, one of the most important of which is mindfulness [12].

\section{* Corresponding Author:}

Seyedeh Batool Hasanpoor-Azghady

Address: Department of Midwifery and Reproductive Health, Nursing Care Research Center, School of Nursing and Midwifery, Iran University of Medical Sciences, Tehran, Iran.

Tel: +98 (915) 5031928

E-Mail: hasanpoorbatool@yahoo.com 
Mindfulness teaches people to accept that thoughts and feelings exist instead of trying to control or suppress their negative thoughts [15]. Higher mindfulness is associated with lower body shaming and can affect the quality of life [17]. People who are more mindful are less likely to compare their bodies to others [18]. Recent studies have shown that mindfulness-based interventions can be beneficial along with routine medical procedures in women with PCOS. Therefore, before dealing with these interventions, it is necessary to find the related factors in different communities to design and formulate intervention and counseling programs according to them to achieve desired results. In this regard, this study aimed to determine relationship of mindfulness with hyperandrogenic symptoms and demographic and fertility factors in women with PCOS.

\section{Materials and Methods}

This is a descriptive correlational study conducted in 2018 on 181 women with PCOS referred to Firoozabadi and Firoozgar hospitals in Tehran, Iran who were selected by continuous sampling method. Data collection tool were 4 questionnaires including a demographic and fertility form, the modified Ferriman-Gallwey Scale for evaluating hirsutism [20], Ludwig Hair Loss Scale [21], and Mindfulness Attention Awareness Scale (MAAS) [22]. MAAS consisted of 15 items rated on a 6-point Likert scale from 1: Almost always to 6: Almost never. The total score ranges from 15 to 90, where higher scores indicate higher mindfulness. Data were analyzed using independent t-test, one-way ANOVA, Kruskal-Wallis test, Pearson correlation test and multiple linear regression analysis in SPSS v. 22 software. Significance level in all tests was considered as $\mathrm{P}<0.05$.

\section{Results}

The mean age of participants was $28.04 \pm 6.42$ ranged from 18 to 46 years. $45.3 \%$ of them had a bachelor's degree or higher; $68.2 \%$ of married participants were infertile and $31.8 \%$ were fertile. The mean of MAAS score was 68.61 \pm 9.88 . Among the demographic and fertility variables (age, duration of marriage, body mass index or BMI, education and occupation of wife and husband, economic status, satisfaction of husband with the body and appearance of wife, marital and fertility status, parity, history of abortion, drug use) and hyperandrogenic symptoms (acne, alopecia and hirsutism), only the variables of age, duration of marriage, BMI, wife's education and occupation, economic status, and husband's satisfaction with wife's body and appearance had a statistically significant relationship with mindfulness. These variables were entered into the linear regression model by Enter method. The marriage duration variable remained in the model, which predicted $22 \%$ of the variance in overall mindfulness score.

\section{Conclusion}

In our study, the MAAS mean score of women was $68.61 \pm 9.88$ which was higher than the median score (52.5). In various studies [24-26], the mean of MAAS score was lower than the median score compared to the present study, which may be related to the higher BMI of the subjects in their study compared to our study. In the present study, there was a significant negative relationship between mindfulness and age. This is against the results of some studies $[27,28]$ where the mindfulness of older adults was higher than that of younger adults [28]. This discrepancy may be due to the lower age of participants in our study and the increase in symptoms of PCOS with the increase of age. In our study, there was a significant negative relationship between marriage duration and mindfulness, which was consistent with the results of Raja-Khan et al. [25] and Daubenmier et al. [29].

Moreover, there was a significant negative relationship between mindfulness and BMI in our study. This is consistent with the results of Katterman et al. [19] and Dalen et al. [30]. In the present study, participants with unfavorable economic status had lower mindfulness score than those with a relatively favorable status, which was consistent with the results of Jensen et al. [32]. Our results showed that participants with very satisfied husbands had a higher mindfulness score than those with indifferent and unsatisfied husbands. This is consistent with results of Burpee et al. [33]. It has been reported that high mindfulness can increase marital satisfaction by improving marital attachment [34]. In our study, the mean MAAS score was not significantly different in terms of the severity of acne, alopecia and hirsutism, which was contrary to the results of Montgomery et al. This discrepancy may be due to the low number of participants in our study with moderate and severe symptoms of acne, alopecia and hirsutism.

In overall, mindfulness was related to age, education and occupation of wife, economic status, husband satisfaction with wife's body and appearance, BMI, and duration of marriage. Among these variables, only marriage duration was able to predict mindfulness in women. The consideration of these related variables in preparation and development of counseling or educational programs along with other treatments for women with PCOS may lead to better results. 


\section{Ethical Considerations}

Compliance with ethical guidelines

This study was obtained its ethical approval from the Ethics Committee of Iran University of Medical Sciences in Tehran, Iran (Code: IR.IUMS.FMD.REC1396.9413373004). The participants were asked to sign a written informed consent prior to study. They were completely informed of the study purpose and procedures, and were assured of the confidentiality of their information.

Funding

The paper was extracted from the MSc. thesis of the first author, Department of Midwifery and Reproductive Health, Nursing Care Research Center, School of Nursing and Midwifery, Iran University of Medical Sciences.

\section{Authors' contributions}

Conceptualization, methodology, investigation:, Seyedeh Batool Hasanpoor-Azghady, Roghayeh Arbabi Moghaddam, Leila Amiri Farahani; Data analysis: Shima Haghani; Data analysis and interpretation: Roghayeh Arbabi Moghaddam, Seyedeh Batool HasanpoorAzghady, and Shima Haghani; Writing, editing and review: Seyedeh Batool Hasanpoor-Azghady, Roghayeh Arbabi Moghaddam.

\section{Conflicts of interest}

The authors declared no conflict of interest.

\section{Acknowledgements}

The authors would like to thank to the Research Vice Chancellor of the Iran University of Educational Sciences as well as the staffs of Firoozabadi and Firoozgar hospitals and the participants who cooperated with the research team. 


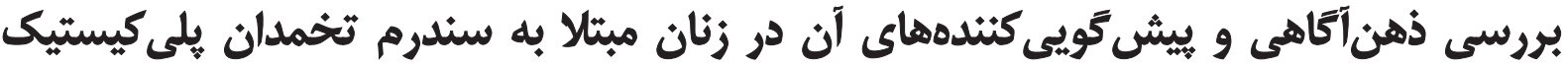

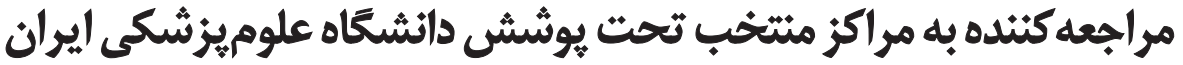

\author{
رقيه اربابى مقدم' هـ، "سيده بتول حسن يور ازغدى' ه، ليلا اميرى فراهانى' (ه، شيما حقانى'
}

ا. مركز تحقيقات مراقبتهاى يرستارى، داثشكله برستارى و مامايعى داتشكاه علوميزشكى خدمات بهـاشتى درمانى ايران، تهران، ايران.

\begin{abstract}
حيكيد
إزمينه سندرم تخمدان يلى كيستيك شايعترين اختالل غدد درونريز زئنان در سنين بارورى است كه افراد رابا مشكلات متعددى از جمله

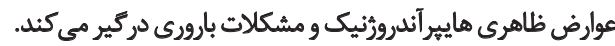

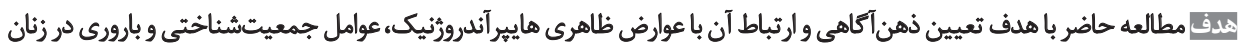

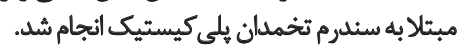

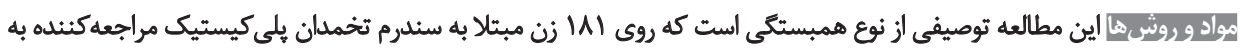

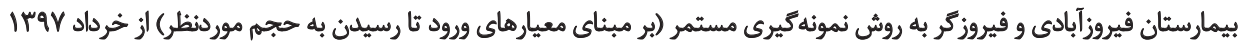

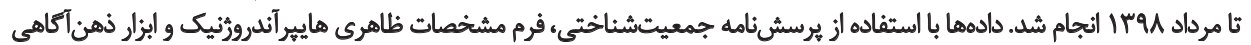

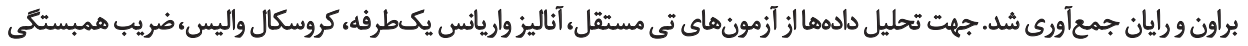

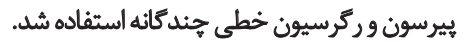

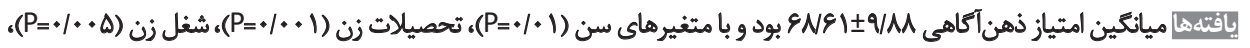

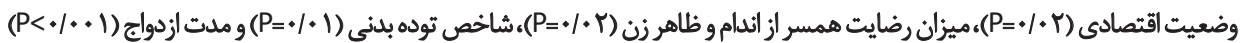

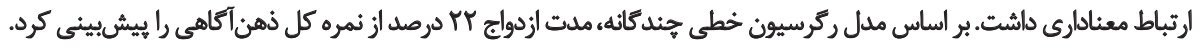

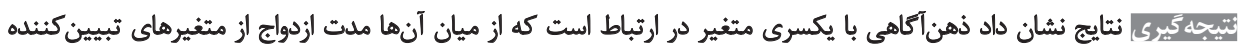

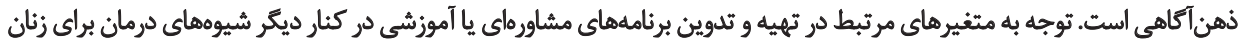

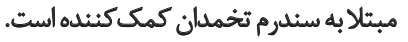

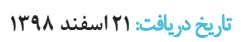

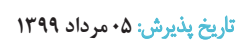

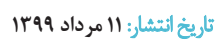

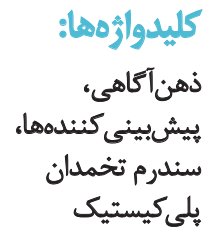

مقدمه

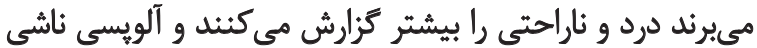

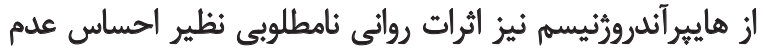

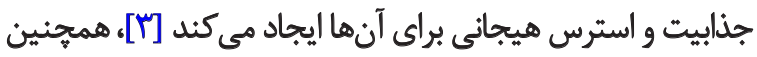

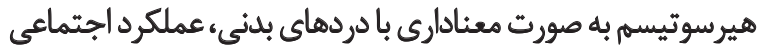

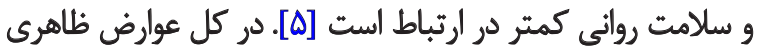

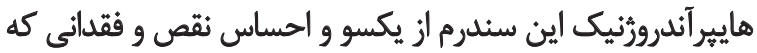

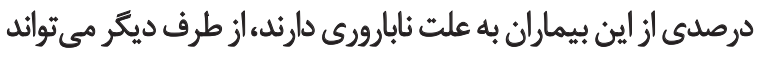

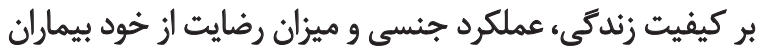

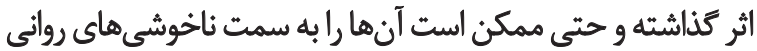

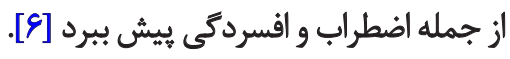

اغلب زنان مبتلا به سندرم تخمدان يلى كيستيك اضافهوزن دارند
سندرم تخمدان بلى كيستيك به عنوان شايعترين اختلال غدد

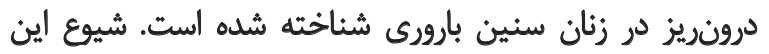

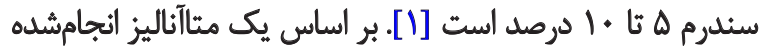

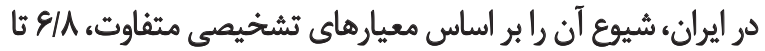

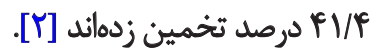

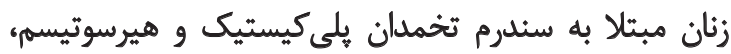

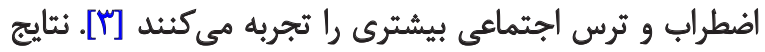

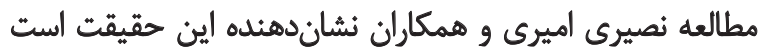

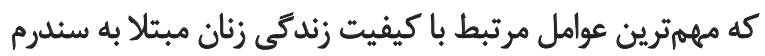

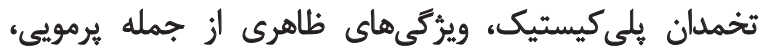

$$
\begin{aligned}
& \text { * : نويسينده مسيئول: }
\end{aligned}
$$

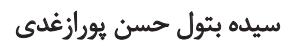

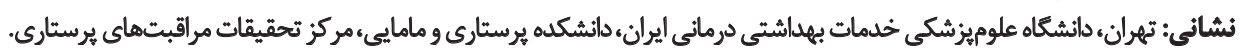

$$
\begin{aligned}
& \text { تلقي: } \\
& \text { راياثامه: hasanpoorbatool@yahoo.com }
\end{aligned}
$$




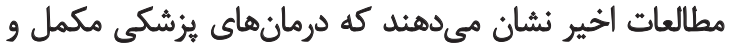

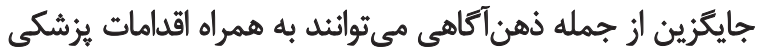

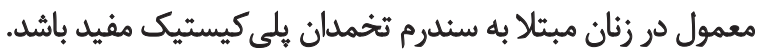

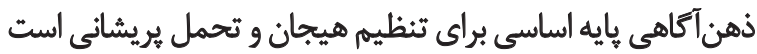

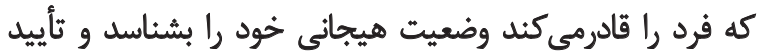

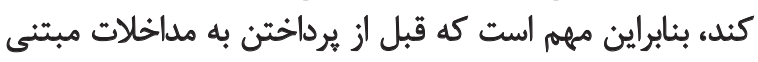

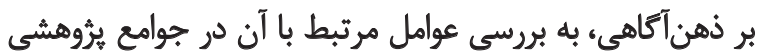

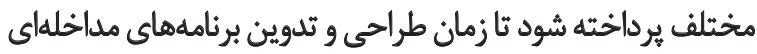

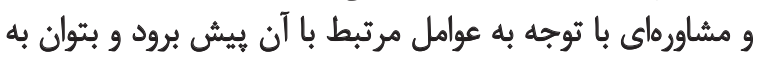

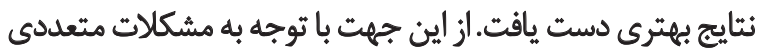

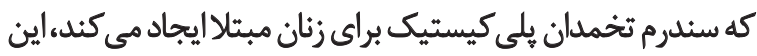

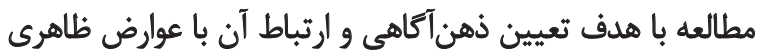

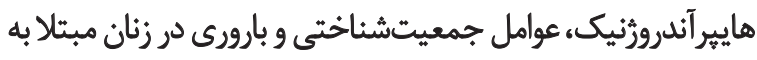

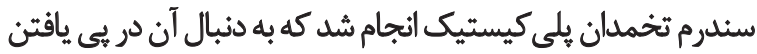
مهمترين عوامل بيشينينى كنينده بوديم.

\section{مواد وروشها}

اين ثرؤش توصيفى از نوع همبستَى است كه از خرداد

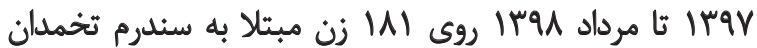

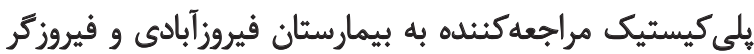

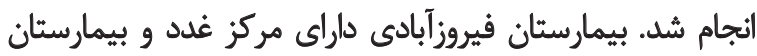

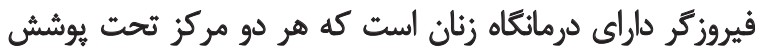

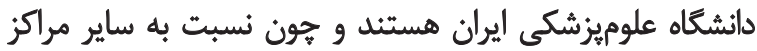

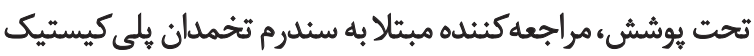

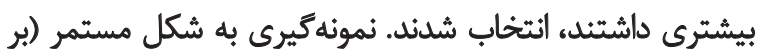

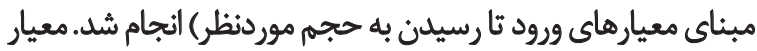

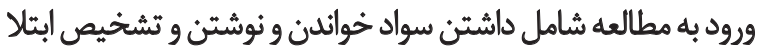

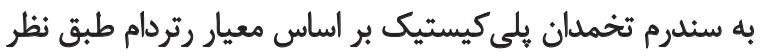

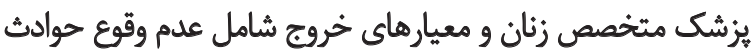

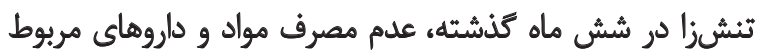

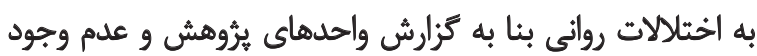
بيمارى هاى سيستميك مزمن بود.

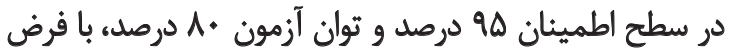

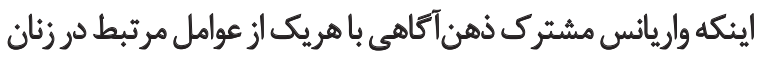

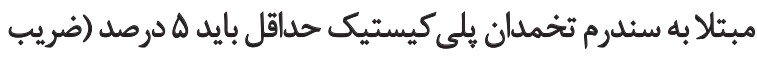

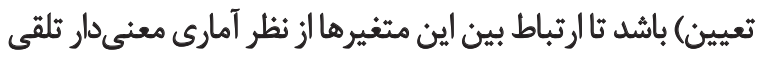
شود، حجم نمونه ال|| نفر برآورد شد.

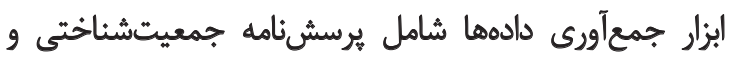

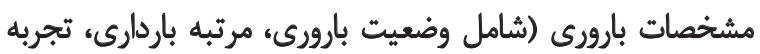

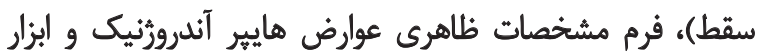

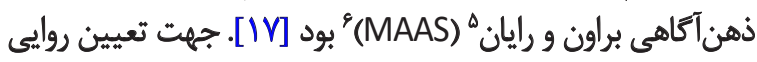

5. Brown, \& Ryan

6. Mindful Attention Awareness Scale
كه مىتواند به دلايل مختلف از جمله سندرم متابوليك، اختلالات

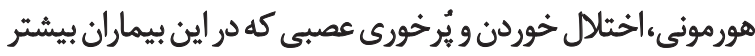

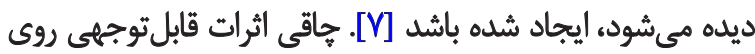

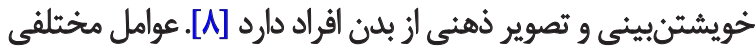

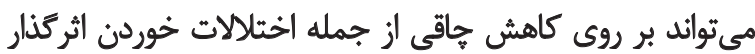

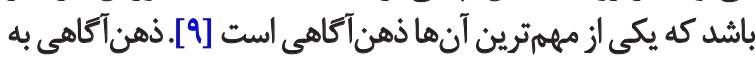

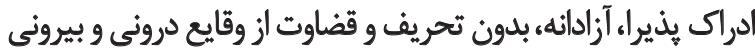

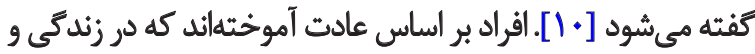

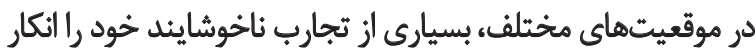

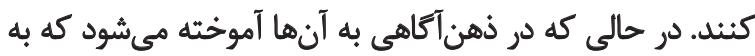

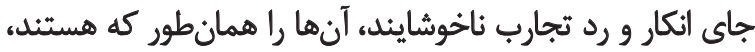

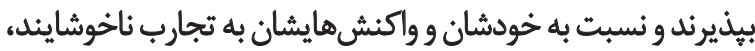

أكاهي داشتئه باشُشند [11]

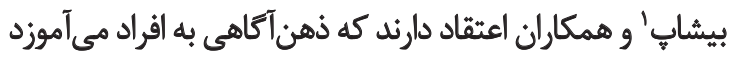

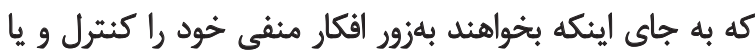

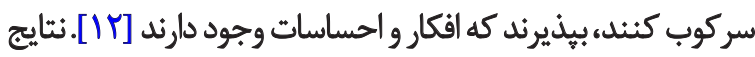

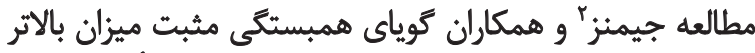

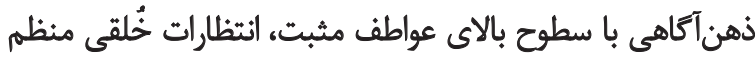

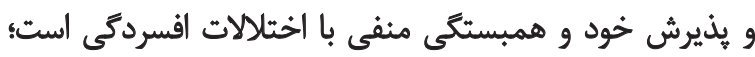

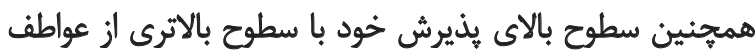

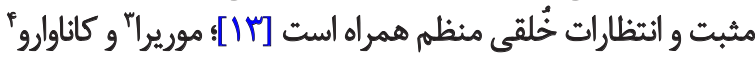

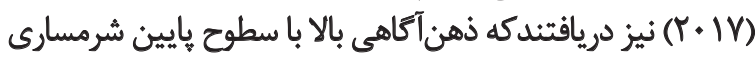

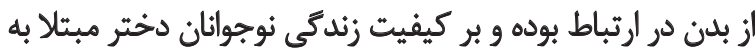

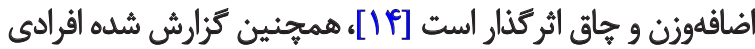

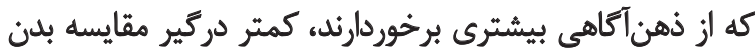

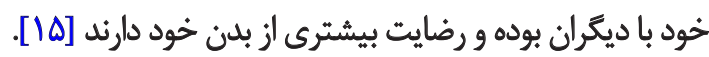
نتايج يك مطالعه توصيفى با هدف بررسى ارتباط بين

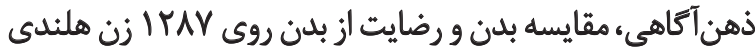

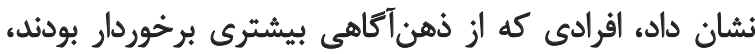

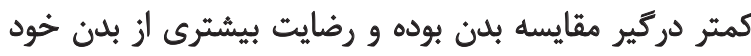

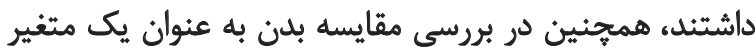

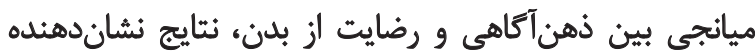

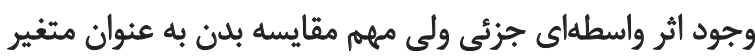

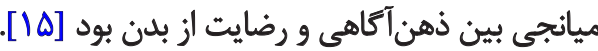

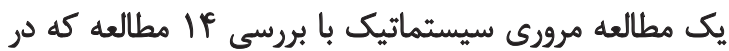

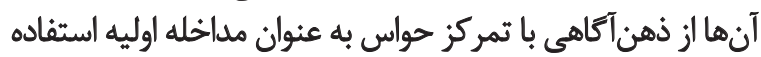

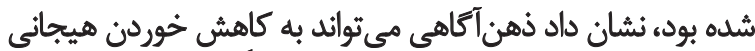

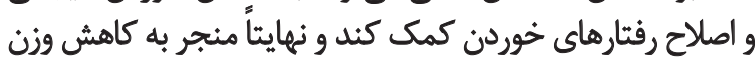

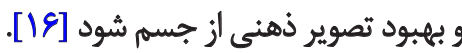

1. Bishop

2. Jimenez

3. Moreira

4. Canavarro 
بس از تأييديه كميته اخلاق و دريافت مجوز نمونهكيرى از إز

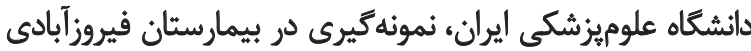

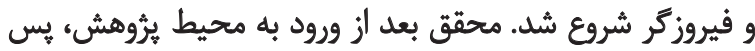

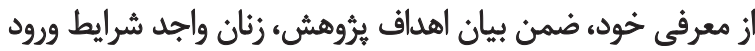

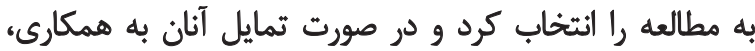

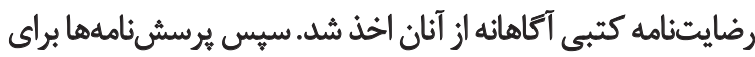

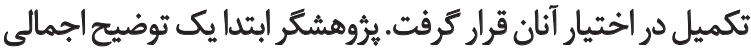

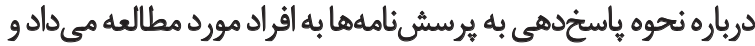

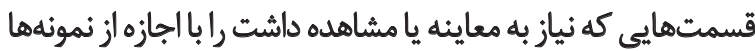

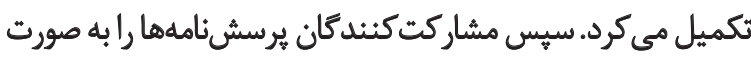

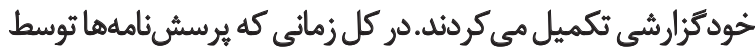

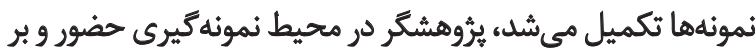

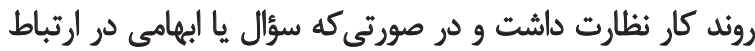

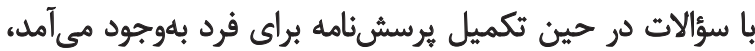

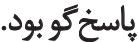

تجزيه و تحليل دادها با استفاده از آزمون هاى آمارى تى مستقل،

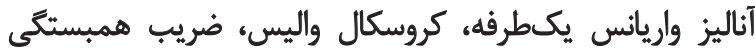

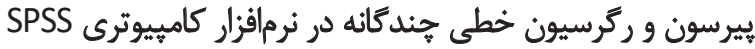

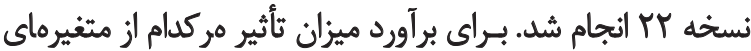

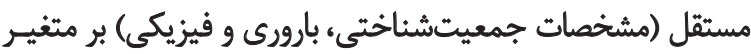

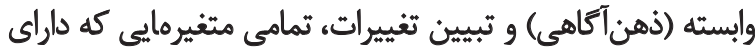

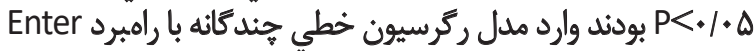

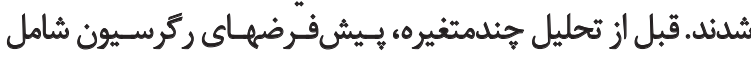

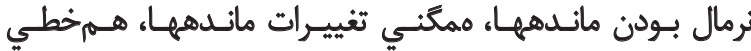

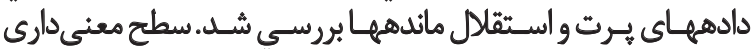
در كليه آزمونها كمتر از هـ ٪ د در نظر كرفته شد.

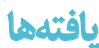

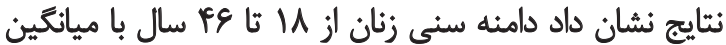

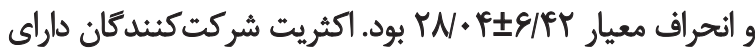

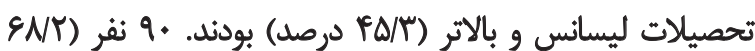

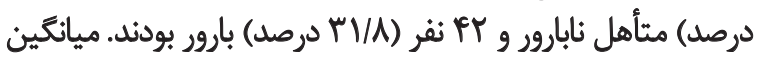

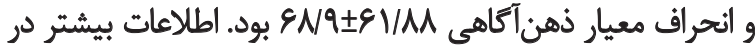

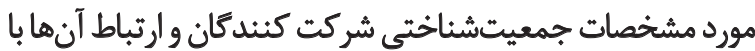
متغير ذهن آكاهى در جدول شماره آمشت آمده است.

از ميان متغيرهايى كه در مطالعه، ارتباط ثكتك آنان باديا

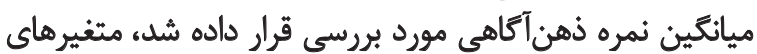

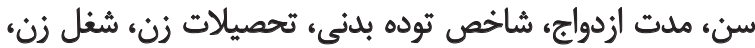

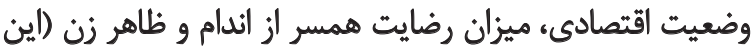

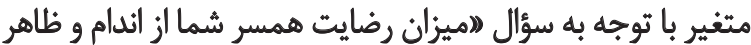

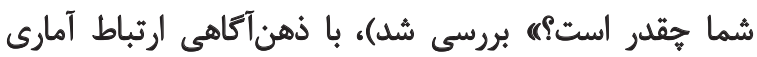

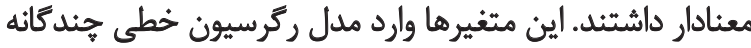
با راهبرد Enter شدند. متغير مدت ازدواج در مدل مدل باقى ماندي
يرسشنامه جمعيتشناختى و مشخصات بارورى، از روايى صورى و

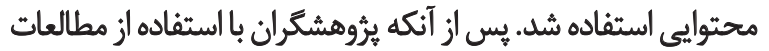

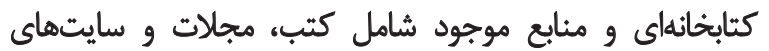

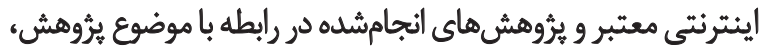

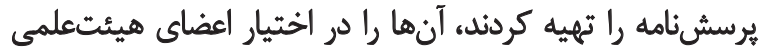

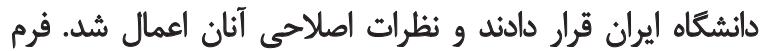

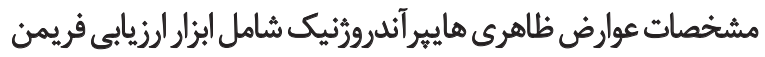

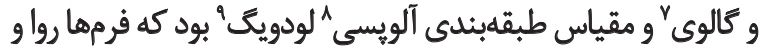

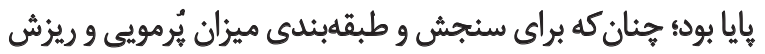

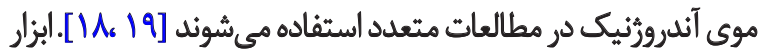

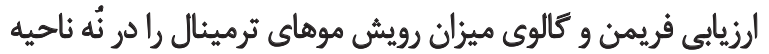

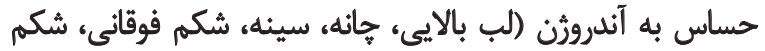

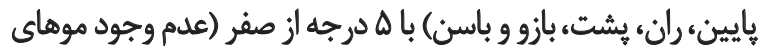

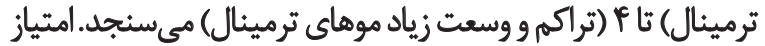

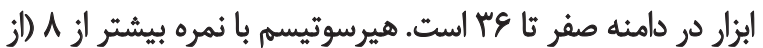

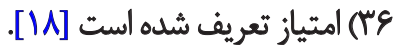
در ايران اكبرزاده و همكاران امتياز صفر تا ع را بدون هيرسوتيسم،

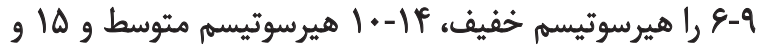

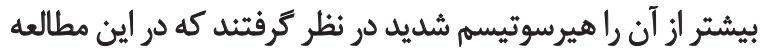

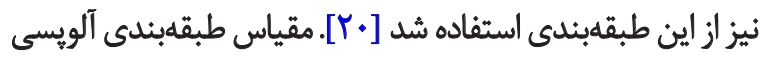

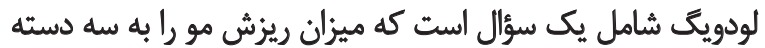

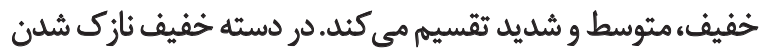

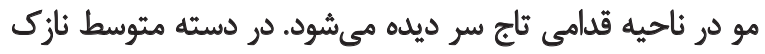

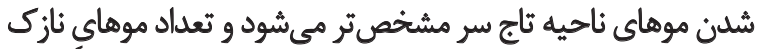

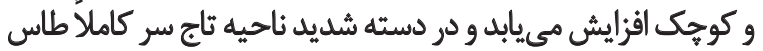

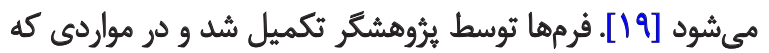

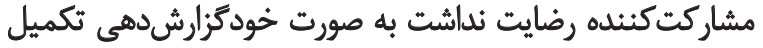

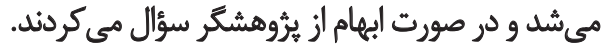

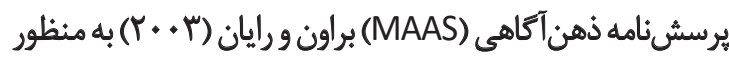
سنجش سطح هوشيارى و توجه نسبت به رويد ريدادها و تجارب جاري جارى

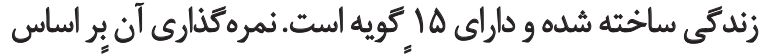

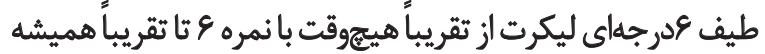

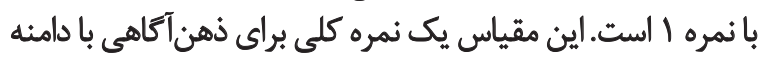

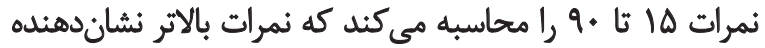

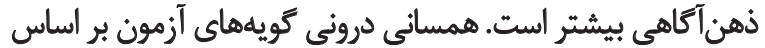

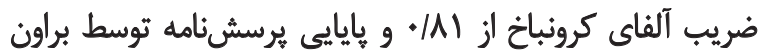

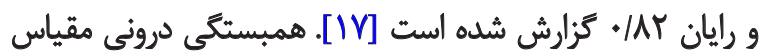

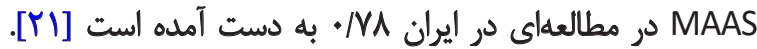

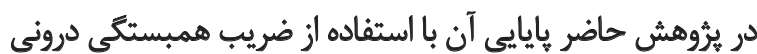

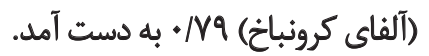

7. Ferriman and galvey

8. Alopecia

9. ludwig 


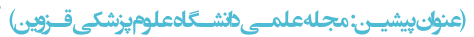

جدول ا. ارتباط مشخصات جمعيتشناختى، بارورى و عوارض ظاهرى هايير آندرورثنيك با ذهن آكاهى در زنان مبتلا به سندرم تخمدان يلى كيستيك (n=|A|)

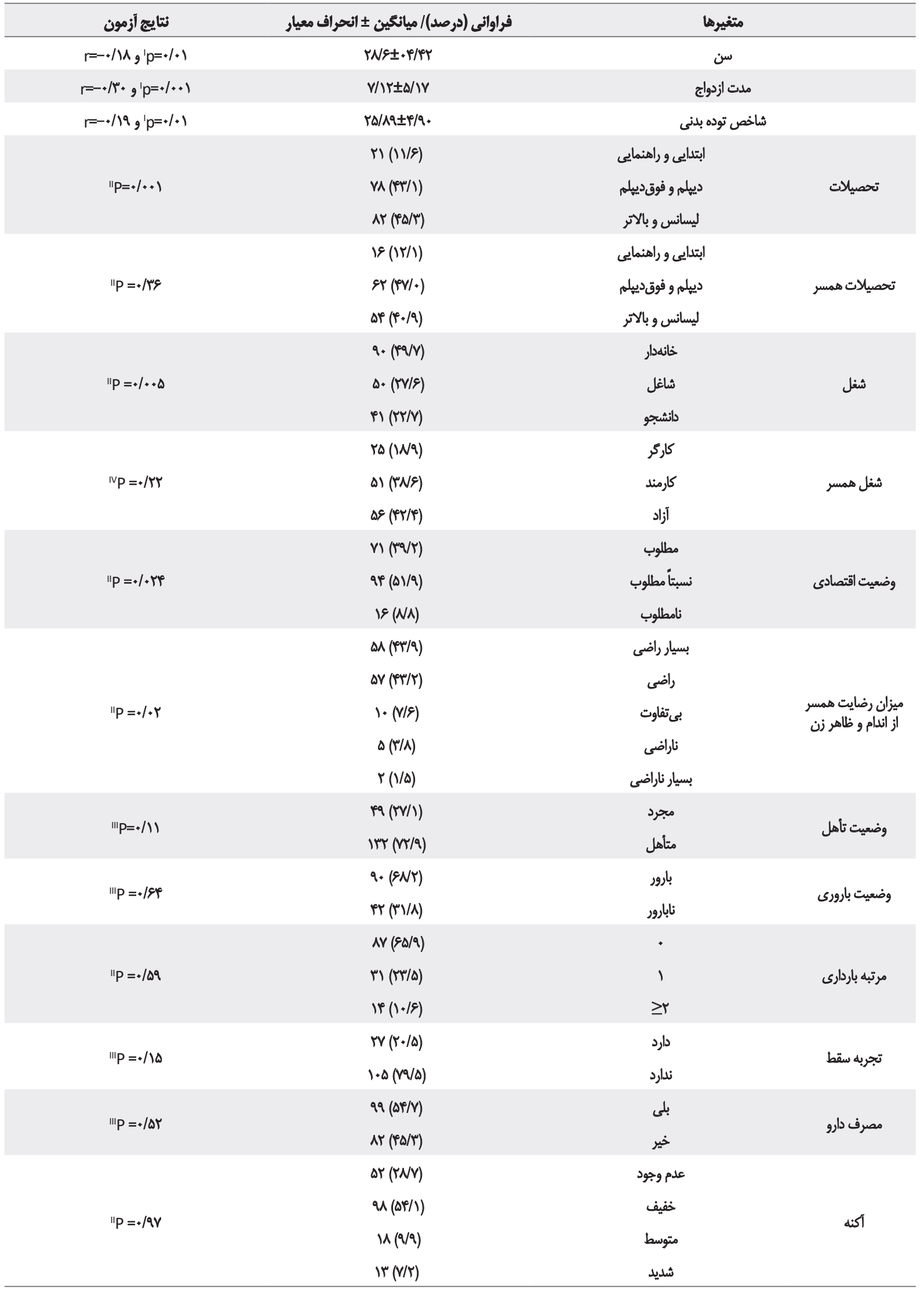




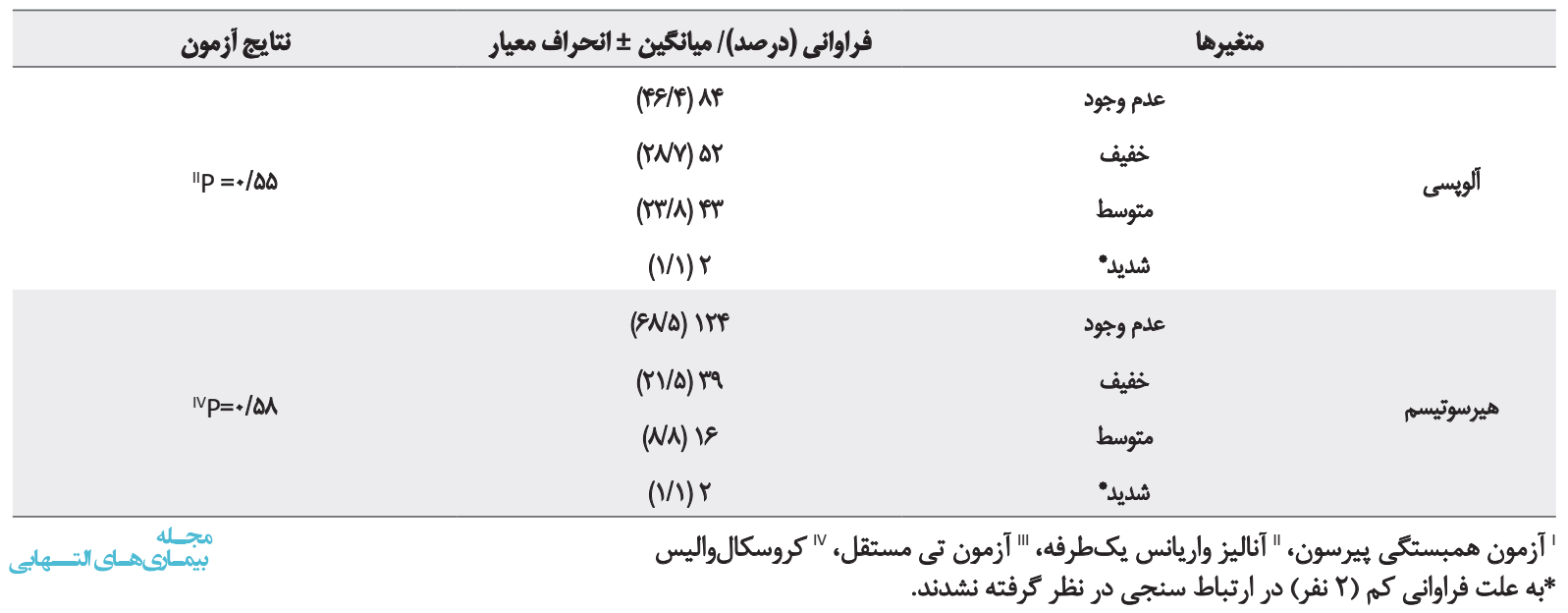

نيز با مطالعه روى زنان مبتلا به اضافهوزن و جاقىى، ميانكين امتياز

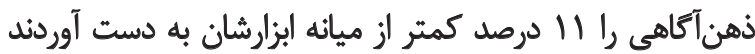

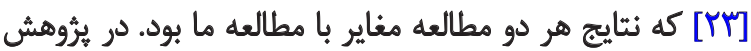

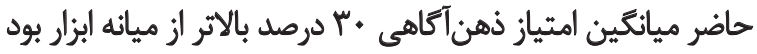

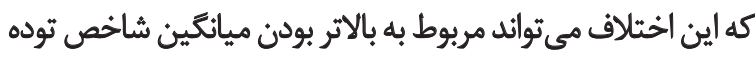

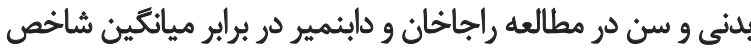
توده بدني و سن در مطالعه ما باشد.

بين ميانكين نمره ذهن آكاهى و سن، ارتباط معنى دار منفى

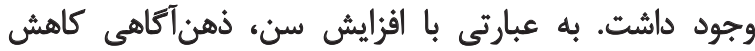

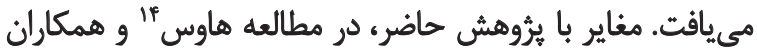

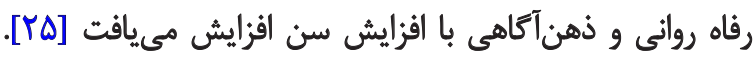

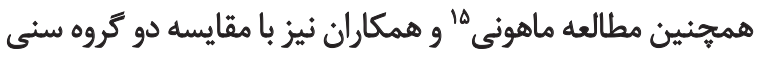

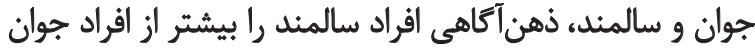

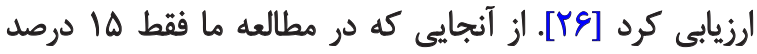

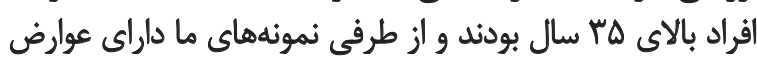

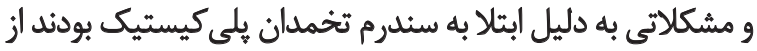

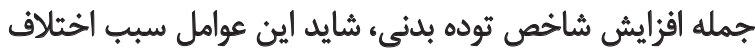
نتايج مطالعه ما با ديكر مطالعات شده است. بين مدت ازدواج و ذهن آكاهى رابطه معنى دار منفى وجود داشت.

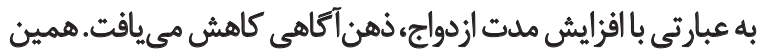

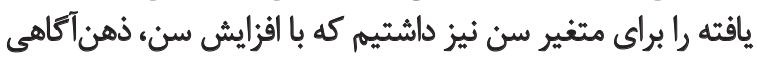

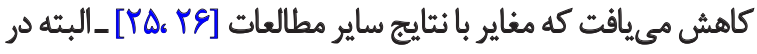

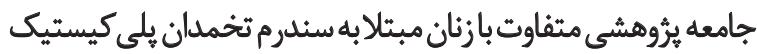

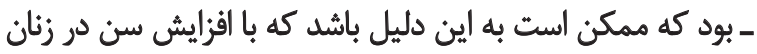

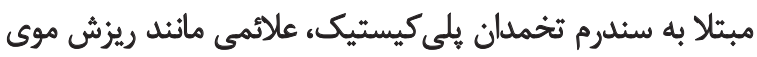

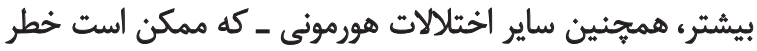

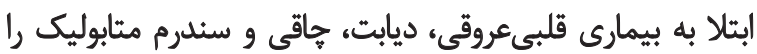

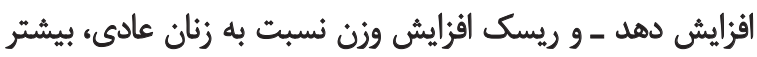

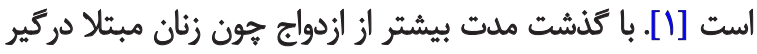

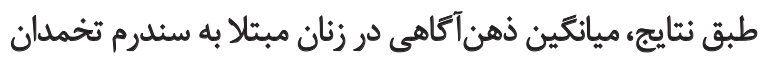

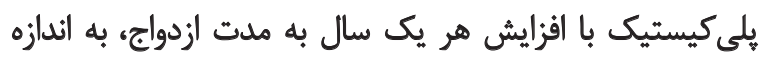

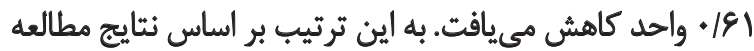

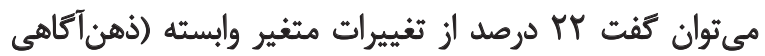

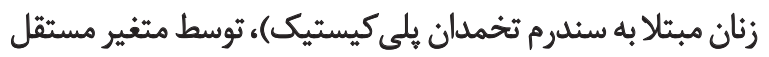

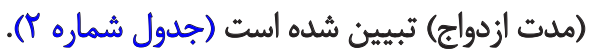

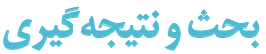

تنتايج اين مطالعه نشان داد امتياز ذهن أكاهي با متغيرهاي سن،

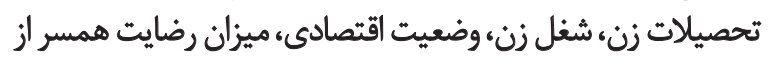

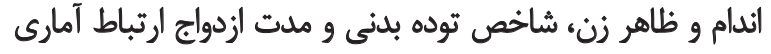

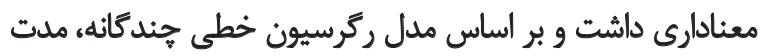

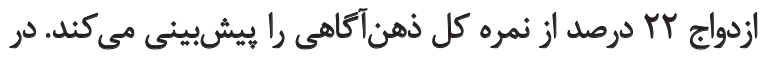

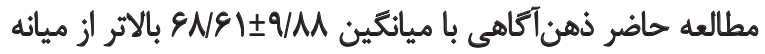

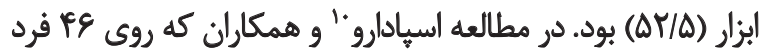

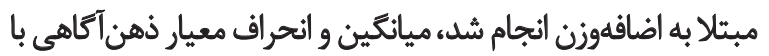

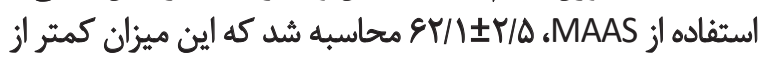

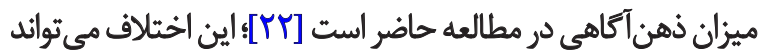

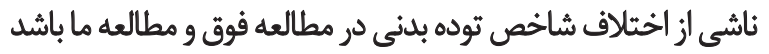

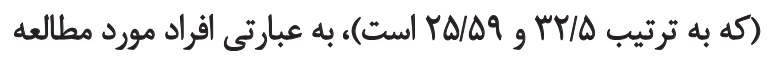

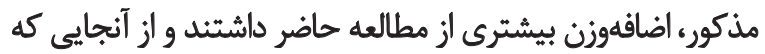

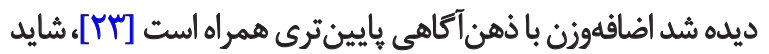

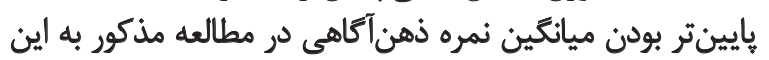
علت باشد.

در مطالعه راجاخان" و همكاران كه روى ع^زن مبتابلابهاضافهوزن

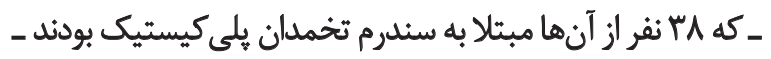

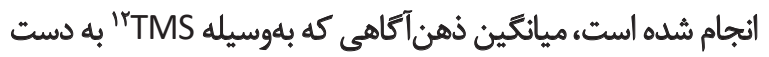

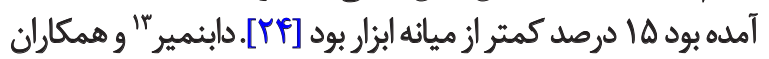

\section{Spadaro}

11. Raja-Khan

12. Toronto Mindfulness Scale

13. Daubenmier 
جدول ب. ييشينى كننده نمره ذهن آكاهى زنان مبتلا به سندرم تخمدان يلى كيستيك (n=|11)

\begin{tabular}{|c|c|c|c|c|c|c|c|}
\hline $\mathrm{R}^{2}$ & ضريب اطمينان & سطح معنى دارى & آماره & ضريب استاندارد & ضريب B & \multicolumn{2}{|c|}{ مثغيرهاي مستقل } \\
\hline & $\Delta S / / F \leftarrow H A / T A$ & | זומ/. & १/s. &.$/ 19$ &.$/ 1 \Delta$ & \multicolumn{2}{|c|}{ سن } \\
\hline & -1.1.8 & 年/ &.$- / 9 y$ &.$- / n \cdot r$ & ו ו ו/T- & \multicolumn{2}{|l|}{ ابتدايع و راهنمايع } \\
\hline & $-\Delta / / F \in F / M A$ & . $/$ grt &.$- / 198$ &.$- / 99$ &.$- / 9 Y$ & دييلم و فوقدييلم & تحصيلات \\
\hline & & \multicolumn{3}{|c|}{ رده مرجع } & \multicolumn{3}{|c|}{ ليسانس و بالاتر } \\
\hline & - - &.$/ 4$ & V/AS &.$/ 14$ & ה & شاغل & \\
\hline & $-F / 116 N<9$ &.$/ 41$ & $\cdot / V$ & .1 .9 & $r / \pi r$ & داتشجو & شغل زن \\
\hline & & \multicolumn{3}{|c|}{ رده مرجع } & \multicolumn{3}{|c|}{ خانهدار } \\
\hline & -V/RA $E T / / T$ & . & $-1 / / 4$ &.$- / 11$ & $-r / \Delta A$ & ن امطلوب & \\
\hline & $-1 / / q q$ b $\Delta / q_{0}$ & $\cdot / \pi \Delta \Delta$ & bir &.$/ 1$ & $r / . \Delta$ & نسيتاً مطلوب & وضعيث اقتصادى \\
\hline & & \multicolumn{3}{|c|}{ رده مرجع } & \multicolumn{3}{|c|}{ هملوب } \\
\hline.$/ T r$ & $-1 / 1 \% b-.0 / .9$ &.$/ . r 1$ & $-r / T$ &.$- / M$ & -.181 & \multicolumn{2}{|c|}{ مدت ازددواج } \\
\hline & 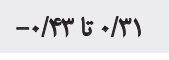 & . Mer &.$- / T T$ & $-.1 . r$ & -.1 .9 & \multicolumn{2}{|c|}{ شاخص توده بدنى } \\
\hline & & \multicolumn{3}{|c|}{ رده مرجع } & \multicolumn{3}{|c|}{ بسيار راضى } \\
\hline & $-8 / \Lambda \cdot t \cdot \pi / \pi f$ &.$\% \vee$ & $-1 / 9$ & $-\cdot N$ & 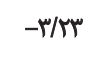 & راضى & \multirow{3}{*}{ ظظرر همسر درياره } \\
\hline & $-11 / / 1 / 4$. &.$/ M A$ & $-1 / \Delta r$ & ז & $-ه / 11$ & بىتفاوت & \\
\hline & $-1 \% / q \cdot b v / r q$ & $+/ 1+1$ & $-1 / 9 T$ &.$- / 1 f$ & $-9 / \pi \Delta$ & ناراضي & \\
\hline
\end{tabular}

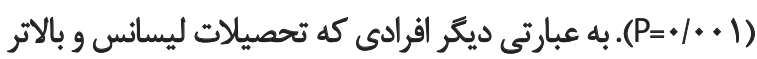

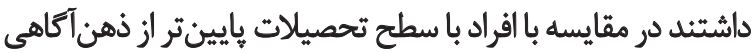

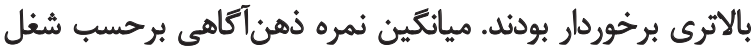

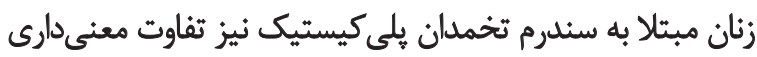

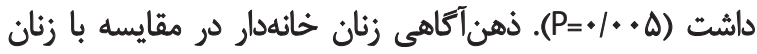

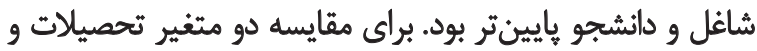

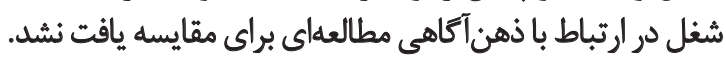

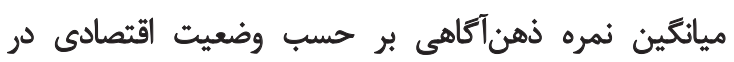

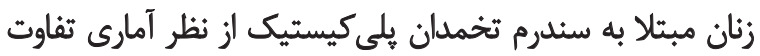

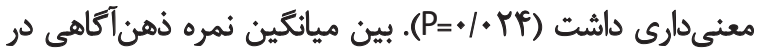

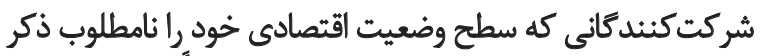

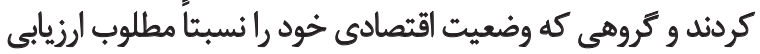

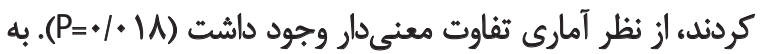

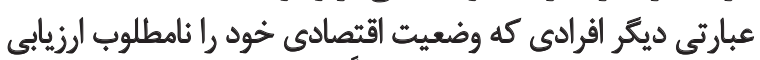

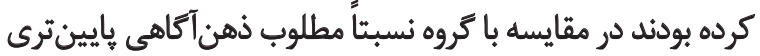

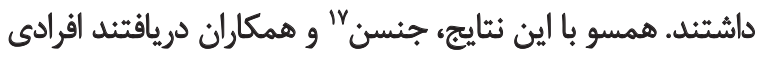

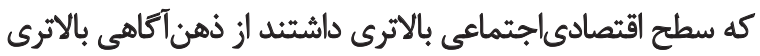

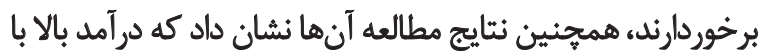

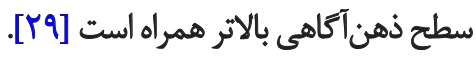
ميانتين ذهن آكاهى بر حسب ميزان رضايت همسر از اندام و ظاهر
عوارض بيشترى مىشوند، شايد اين موضوع ارتباط معكوس مدت

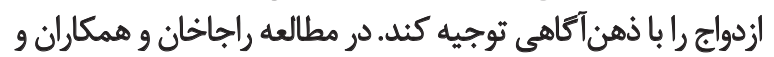

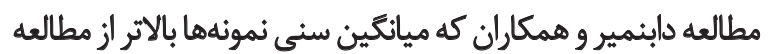

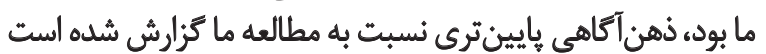

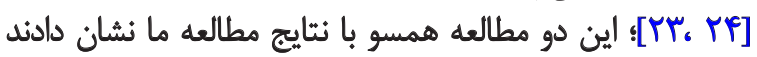

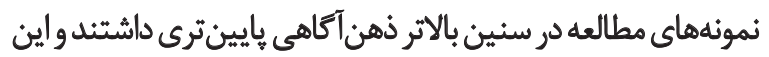
افراد نيز از شاخص توده بدنى بالاترى نيز برخوردار بودندين

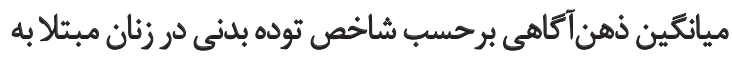

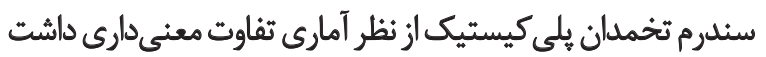

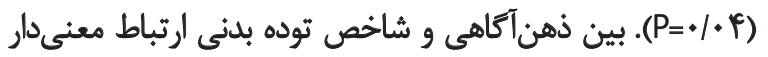

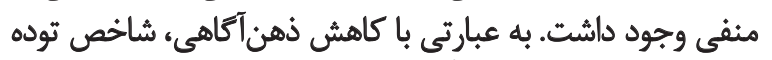

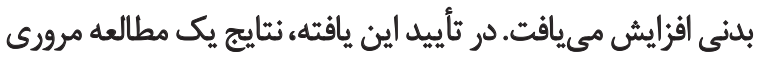

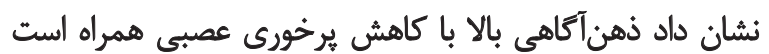

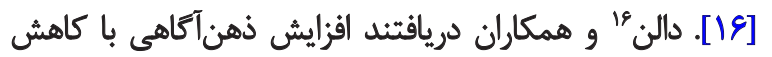

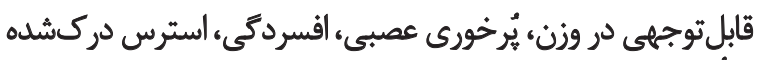

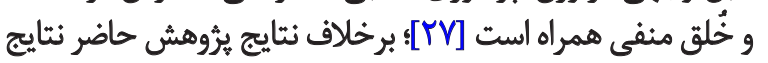

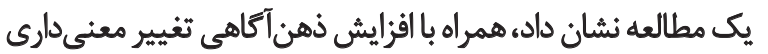

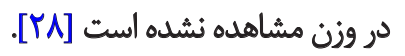

ميانكين نمره ذهن آكاهى بر حسب تحصيلات زنان مبتئلا به مانه

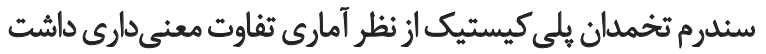

16. Dalen 


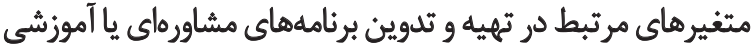

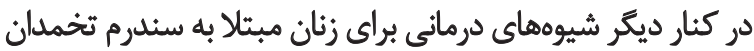
ممكن است نتايج بهترى دربر داشته باشد درئ

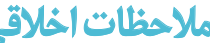

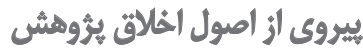

بعد از توضيح اهداف مطالعه و اطمينان درباره محرمانه ماندن

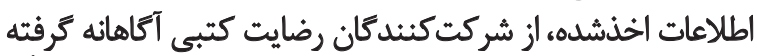

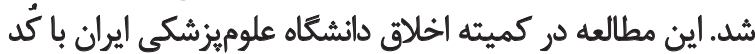

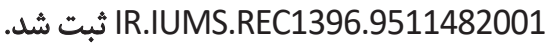

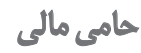

اين مقاله مستخرج از ياياننامه كارشناسىارشد نويسنده اول، در ئري

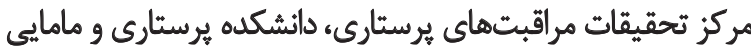

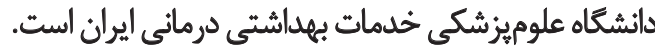

$$
\text { مشاركت نويسندكان }
$$

مفهوم سازى و روش شناسى، تحقيق و بررسى: سيده بتول حسن

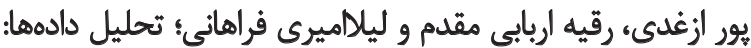

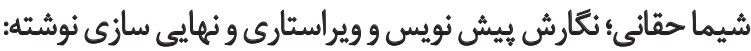

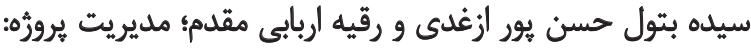

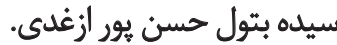

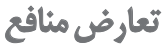

نويسندكان اعلام مى دارند اين مقاله تعارض منافع ندارد.

$$
\text { تشكر و قدردانى ته }
$$

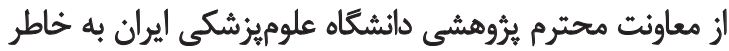

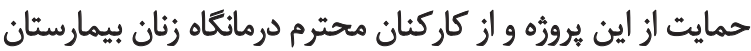

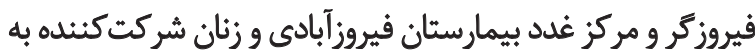
خاطر همكارى با تروه تحقيق سياسكزاريم. فيرون.
فرد در زنان مبتلا به سندرم تخمدان يلى كيستيك از نظر آمارى

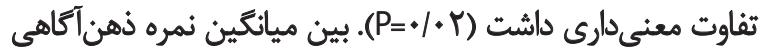

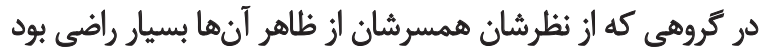

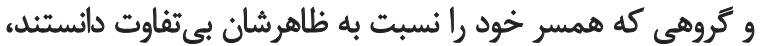

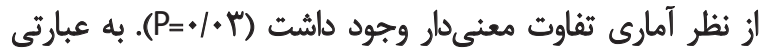

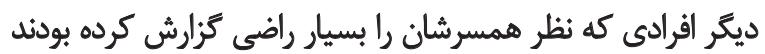

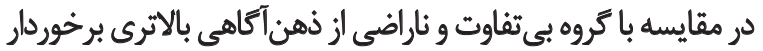

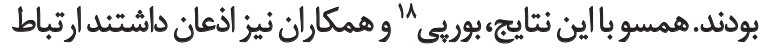

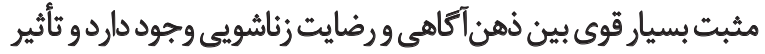

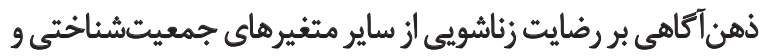

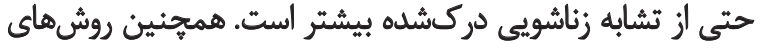

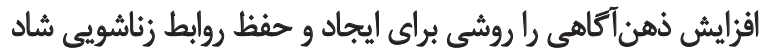

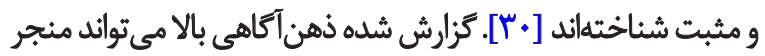

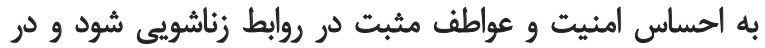

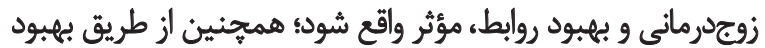

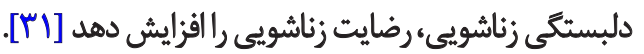

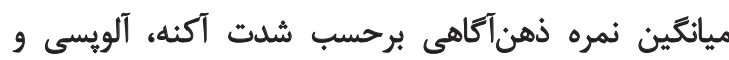

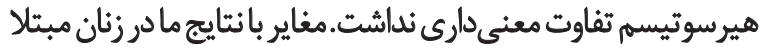

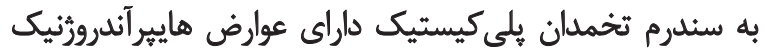

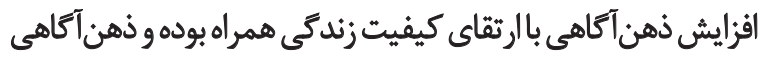

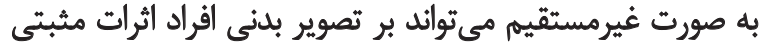

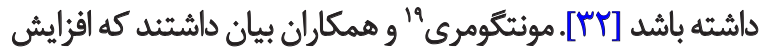

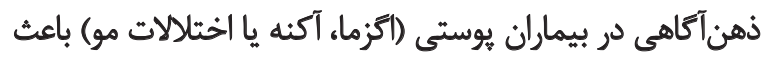

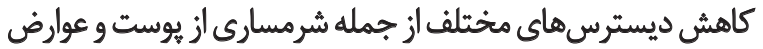

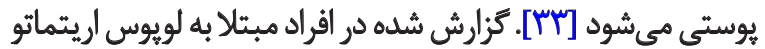

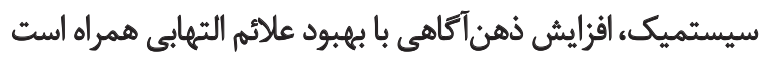

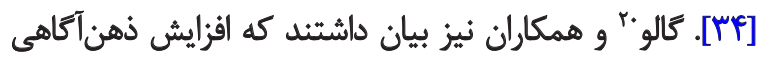

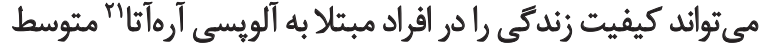

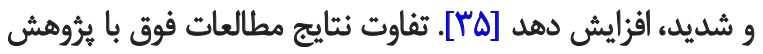

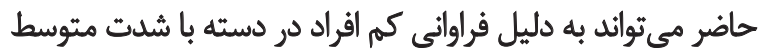

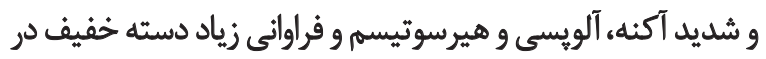

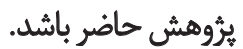

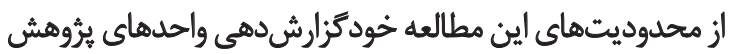

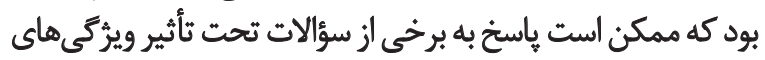

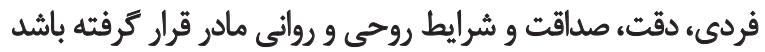
كه كنترل آن خارج أز اختيار بثروهشكر بودي

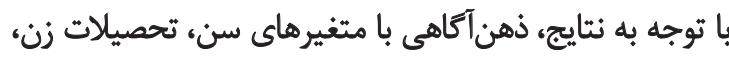

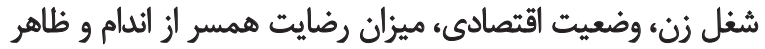

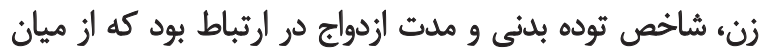

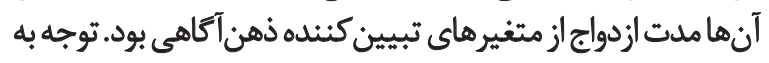

18. Burpee

19. Montgomery

20. Gallo

21. Alopcia areata 


\section{References}

[1] Berek JS. Berek \& Novak's gynecology. $16^{\text {th }}$ edition. Alphen aan den Rijn: Wolters Kluwer; 2019. https://shop.lww.com/Berek--Novak-s-Gynecology/p/9781496380333

[2] Sayehmiri F, Kiani F, Maleki F, Ahmadi M, Shohani M. Prevalence of polycystic ovary syndrome in Iranian women: a systematic review and meta-analysis. Iran J Obstet Gynecol Infertil. 2014; 17(115):11-21.

[3] Janssen OE, Hahn S, Tan S, Benson S, Elsenbruch S. Mood and sexual function in polycystic ovary syndrome. Semin Reprod Med. 2008; 26(1):45-52. [DOI:10.1055/s-2007-992924] [PMID]

[4] Nasiri Amiri F, Ramezani Tehrani FA, Simbar M, Tahamtan Mohammadpour RA. Concerns of women with polycystic ovary syndrome: A qualitative study. IJEM. 2013; 15(1):41-51. [In Persian]http://ijem.sbmu.ac.ir/article-1-1390-en.html

[5] Ekback MP, Lindberg M, Benzein E, Arestedt K. Social support: An important factor for quality of life in women with hirsutism. Health Qual Life Outcomes. 2014; 12:183. [DOI:10.1186/ s12955-014-0183-3] [PMID] [PMCID]

[6] Barry JA, Kuczmierczyk AR, Hardiman PJ. Anxiety and depression in polycystic ovary syndrome: a systematic review and meta-analysis. Hum Reprod. 2011; 26(9):2442-51. [DOI:10.1093/ humrep/der197] [PMID]

[7] Morosi A, Jeanes Y. Food cravings, binge eating and emotional eating behaviours in overweight and obese women with polycystic ovary syndrome. Proc Nutr Soc. 2017; 76(OCE1):e15. [DOI:10.1017/S0029665117000155]

[8] Kinzl JF. Obesity: Stigmatization, discrimination, body image. Wien Med Wochenschr. 2016; 166(3-4):117-20. [DOI:10.1007/ s10354-016-0443-4] [PMID]

[9] Atkinson MJ, Wade TD. Does mindfulness have potential in eating disorders prevention? A preliminary controlled trial with young adult women. Early interv psychiatry. 2016; 10(3):23445. [DOI:10.1111/eip.12160] [PMID]

[10] Brown KW, Ryan RM, Creswell JD. Mindfulness: Theoretical foundations and evidence for its salutary effects. Psychol Inq. 2007; 18(4):211-37. [DOI:10.1080/10478400701598298]

[11] Crane R. Mindfulness-based cognitive therapy: Distinctive features. UK: Routledge; 2009. [DOI:10.4324/9780203882344]

[12] Bishop SR, Lau M, Shapiro S, Carlson L, Anderson ND, Carmody J, et al. Mindfulness: A proposed operational definition. Clin Psychol Sci Pract. 2004; 11(3):230-41. [DOI:10.1093/clipsy.bph077]

[13] Jimenez SS, Niles BL, Park CL. A mindfulness model of affect regulation and depressive symptoms: Positive emotions, mood regulation expectancies, and self-acceptance as regulatory mechanisms. Pers Individ Dif. 2010; 49(6):645-50. [DOI:10.1016/j.paid.2010.05.041]

[14] Moreira H, Canavarro MC. Is body shame a significant mediator of the relationship between mindfulness skills and the quality of life of treatment-seeking children and adolescents with overweight and obesity? Body Image. 2017; 20:49-57. [DOI:10.1016/j.bodyim.2016.11.004] [PMID]
[15] Dijkstra P, Barelds DPH. Examining a model of dispositional mindfulness, body comparison, and body satisfaction. Body Image. 2011; 8(4):419-22. [DOI:10.1016/j.bodyy im.2011.05.007] [PMID]

[16] Katterman SN, Kleinman BM, Hood MM, Nackers LM, Corsica JA. Mindfulness meditation as an intervention for binge eating, emotional eating, and weight loss: A systematic review. Eat Behav. 2014; 15(2):197-204. [DOI:10.1016/j.eatt beh.2014.01.005] [PMID]

[17] Brown KW, Ryan RM. The benefits of being present: Mindfulness and its role in psychological well-being. J Pers Soc Psychol. 2003; 84(4):822-48. [DOI:10.1037/0022-3514.84.4.822] [PMID]

[18] Ferriman D, Gallwey JD. Clinical assessment of body hair growth in women. J Clin Endocrinol Metab. 1961; 21:1440-7. [DOI:10.1210/jcem-21-11-1440] [PMID]

[19] Ludwig E. Classification of the types of androgenetic alopecia (common baldness) occurring in the female sex. $\mathrm{Br} J$ Dermato. 1977; 97(3):247-54. [DOI:10.1111/j.1365-2133.1977. tb15179.x] [PMID]

[20] Akbarzadeh M, Naderi T, Dabaghmanesh MH, Tabatabaie $\mathrm{H}_{\text {, }}$ Zare Z. The prevalence of hirsutism in girls 14 to 18 years old and its relationship with polycystic ovary syndrome in Shiraz, Iran. IJOGA. 2014; 16(81):8-15. [In Persian] https://ijogi.mums. ac.ir/article_2232.html?lang=en

[21] Ghasemipour Y, Ghorbani N. Mindfulness and basic psychological needs among patients with coronary heart disease. Iran J Psychiatry Clin Psychol. 2010; 16(2):154-62. [In Persian] http://ijpcp.iums.ac.ir/article-1-1047-en.html

[22] Spadaro KC, Davis KK, Sereika SM, Gibbs BB, Jakicic JM, Cohen $\mathrm{SM}$. Effect of mindfulness meditation on short-term weight loss and eating behaviors in overweight and obese adults: A randomized controlled trial. J Complement Integr Med. 2017; 15(2). [DOI:10.1515/jcim-2016-0048] [PMID]

[23] Daubenmier J, Kristeller J, Hecht FM, Maninger N, Kuwata $M$, Jhaveri $K$, et al. Mindfulness intervention for stress eating to reduce cortisol and abdominal fat among overweight and obese women: An exploratory randomized controlled study. J Obes. 2011; 2011(2090-0708):651936. [DOI:10.1155/2011/651936] [PMID] [PMCID]

[24] Raja-Khan N, Agito K, Shah J, Stetter CM, Gustafson TS, Socolow $\mathrm{H}$, et al. Mindfulness-based stress reduction for overweight/obese women with and without polycystic ovary syndrome: Design and methods of a pilot randomized controlled trial. Contemp Clin Trials. 2015; 41:287-97. [DOI:10.1016/j. cct.2015.01.021] [PMID] [PMCID]

[25] Hohaus LC, Spark J. 2672-Getting better with age: Do mindfulness \& psychological well-being improve in old age? Eur Psychiat. 2013; 28(S1):1. [DOI:10.1016/S0924-9338(13)77295-X]

[26] Mahoney CT, Segal DL, Coolidge FL. Anxiety sensitiv ity, experiential avoidance, and mindfulness among younger and older adults: Age differences in risk factors for anxiety symptoms. Int J Aging Hum Dev. 2015; 81(4):217-40. [DOI:10.1177/0091415015621309] [PMID] 
[27] Dalen J, Smith BW, Shelley BM, Sloan AL, Leahigh L, Begay D. Pilot study: Mindful Eating and Living (MEAL): Weight, eating behavior, and psychological outcomes associated with a mindfulness-based intervention for people with obesity. Complement Ther Med. 2010; 18(6):260-4. [DOI:10.1016/j. ctim.2010.09.008] [PMID]

[28] Fuller NR, Sainsbury A, Caterson ID, Enarsson M, Denyer G, Manns $C$, et al. Examining mindfulness as a predictor of weight loss - Findings from the DIABEGG study. Obes Res Clin Pract. 2017; 11(1):88-96. [DOI:10.1016/j.orcp.2016.03.004] [PMID]

[29] Jensen CG, Krogh SC, Westphael G, Hjordt LV. Mindfulness is positively related to socioeconomic job status and income and independently predicts mental distress in a long-term perspective: Danish validation studies of the Five-Factor Mindfulness Questionnaire. Psychol Assess. 2019; 31(1):e1-20. [DOI:10.1037/pas0000667] [PMID]

[30] Burpee LC, Langer EJ. Mindfulness and marital satisfaction. J Adult Dev. 2005; 12(1):43-51. [DOI:10.1007/s10804-005-12816]

[31] Jones KC, Welton SR, Oliver TC, Thoburn JW. Mindfulness, spousal attachment, and marital satisfaction: A mediated model. J Fam. 2011; 19(4):357-61. [DOI:10.1177/1066480711417234]

[32] Morgan JF, Lazarova S, Schelhase M, Saeidi S. Ten session body image therapy: Efficacy of a manualised body image therapy. Eur Eat Disord Rev. 2014; 22(1):66-71. [DOI:10.1002/erv.2249] [PMID]

[33] Montgomery K, Norman P, Messenger AG, Thompson AR. The importance of mindfulness in psychosocial distress and quality of life in dermatology patients. Br J Dermat. 2016; 175(5):930-6. [DOI:10.1111/bjd.14719] [PMID] [PMCID]

[34] Shenefelt PD. Mindfulness-based cognitive hypnotherapy and skin disorders. Am J Clin Hypn. 2018; 61(1):34-44. [DOI:10 .1080/00029157.2017.1419457] [PMID]

[35] Gallo R, Chiorri C, Gasparini G, Signori A, Burroni A, Parodi A. Can mindfulness-based interventions improve the quality of life of patients with moderate/severe alopecia areata? A prospective pilot study. J Am Acad Dermatol. 2017; 76(4):757-9. [DOI:10.1016/j.jaad.2016.10.012] [PMID] 